Published in final edited form as:

Inorg Chem. 2019 August 05; 58(15): 10287-10294. doi:10.1021/acs.inorgchem.9b01441.

\title{
Structures and Spectroscopic Properties of Metallocorrole Nanoparticles
}

\author{
Julius Oppenheim ${ }^{\dagger}$, Mike H. B. Gray ${ }^{\dagger}$, , Angel J. Di Bilio ${ }^{\dagger}, \bigcirc$, Bradley J. Brennan ${ }^{\dagger}$, Lawrence \\ M. Henling ${ }^{\dagger}$, Punnajit Lim ${ }^{\bigcirc}$, Matan Soll ${ }^{\ddagger}$, John Termini ${ }^{\bigcirc}$, Scott C. Virgil ${ }^{\dagger}$, Zeev Gross ${ }^{\ddagger}$, \\ Harry B. Gray ${ }^{\dagger, *}$ \\ †Beckman Institute, California Institute of Technology, Pasadena, CA, 91125, USA \\ §Department of Chemistry, Occidental College, Los Angeles, CA, 90041, USA \\ 'Department of Molecular Medicine, Beckman Research Institute of the City of Hope, Duarte, CA, \\ 91010, USA \\ ¥Schulich Faculty of Chemistry, Technion-Israel Institute of Technology, Haifa, 32000, Israel
}

\begin{abstract}
In aqueous media, hydrophobic metallocorroles form nanoparticles that are potential theranostic anticancer agents. We have analyzed the electronic and Raman spectra of $\mathrm{Al}(\mathrm{III}), \mathrm{Ga}(\mathrm{III})$, and $\mathrm{Au}(\mathrm{III})$ corrole nanoparticles (and made comparisons with DFT-validated assignments of the IR spectra of corresponding monomers) in order to estimate the strengths of corrole-corrole electronic couplings in these assemblies. We find that these spectra are virtually unchanged upon aggregation, confirming that the intermolecular interactions in these nanoparticles are very weak.
\end{abstract}

\section{Graphical Abstract}

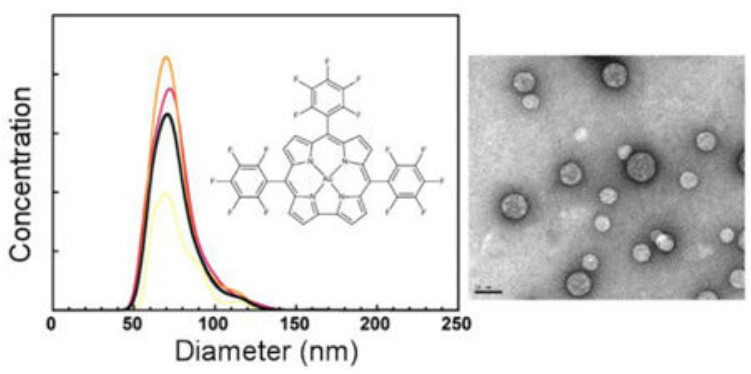

Electronic and vibrational spectroscopic investigations of spheroidal Au-tpfc nanoparticles (TEM image) indicate that corrole-corrole electronic couplings are relatively weak.

\footnotetext{
*Corresponding Author hbgray@caltech.edu. ASSOCIATED CONTENT

Supporting Information

The following files are available free of charge.

Additional information on the Au-tpfc synthesis, XRD and Au-tpfc crystal structure, ICP-MS, fluorescence, NTA, TEM, UV-visible spectra, Raman and IR spectra.

Accession Codes

CCDC 1914233 contains the supplementary crystallographic data for this paper. These data can be obtained free of charge from The Cambridge Crystallographic Data Centre via www.ccdc.cam.ac.uk/structures.
} 


\section{INTRODUCTION}

Recent work has shown that corroles form nanoparticles (NPs) in aqueous media, ${ }^{1}$ much like porphyrins (where aggregates have been investigated since the late 1930s). ${ }^{2-5}$ Two modes of noncovalent interactions have been identified in porphyrin aggregates: side-by-side, or J-

type; and face-to-face, or H-type ${ }^{6}$ Both Soret- and Q-bands in H-type spectra are red-shifted relative to absorptions in the corresponding monomers. Interestingly, these same absorptions shift in different directions in J-assemblies (Soret is blue shifted; Q is red shifted). ${ }^{7}$

As very little is known about the intermolecular electronic couplings in corrole NPs, we have made in-depth experimental and density functional theory (DFT) investigations of the UV-visible, Raman, and IR spectroscopic properties of quasi-soluble aggregates (NPs, as well as monomeric and crystalline reference samples) of $\mathrm{Au}(\mathrm{III}), \mathrm{Ga}(\mathrm{III})$, and $\mathrm{Al}(\mathrm{III})$ complexes of 5,10,15-tris(pentafluorophenyl)corrole (Au-tpfc, Ga-tpfc, Al-tpfc). ${ }^{1,8-10} \mathrm{We}$ also report DFT-validated IR spectroscopic signatures of axial ligand binding in Ga-tpfc and Al-tpfc (Au-tpfc is not axially ligated; see Figure S1). The brightly luminescent Ga(III) and $\mathrm{Al}(\mathrm{III})$ complexes are promising optical imaging agents, whereas the $\mathrm{Au}(\mathrm{III})$ and related complexes have been shown to destroy cancerous tissues. ${ }^{1,11,12}$

\section{RESULTS}

\subsection{Metallocorrole Nanoparticles.}

Nanoparticles were formed by rapid injection of DMSO or pyridine solutions of M(III)-tpfc $(\mathrm{M}=\mathrm{Au}, \mathrm{Ga}, \mathrm{Al})$ complexes into aqueous media. Stable colloidal suspensions were obtained without the use of surfactants. ${ }^{13,14}$ The M(III)-tpfc colloidal suspensions are less optically transparent than solutions of corresponding monomers in pure organic solvents. Notably, the exceptionally bright red photoemissions of monomeric $\mathrm{Ga}(\mathrm{III})$ and $\mathrm{Al}(\mathrm{III})$ corroles are strongly quenched in the aggregates (Figures S2-3). White-light illuminated aggregated samples are bluish purple, owing to light scattering from the broadened Soret band (Figures S2-4). ${ }^{15}$

Nanoparticle Tracking Analysis (NTA) revealed relatively narrow size distributions for Altpfc(py) $)_{2} \mathrm{NPs}$ in $2 \%$ pyridine/water and Ga-tpfc(py) NPs in $2 \% \mathrm{DMSO} /$ water with a median hydrodynamic size of 54-58 $\mathrm{nm}(\mathrm{SD}=16 \mathrm{~nm})$. A much larger degree of polydispersity was obtained for Al-tpfc(py) $)_{2} \mathrm{NPs}$ in $2 \% \mathrm{DMSO} /$ water (a median size of about $90 \mathrm{~nm}, \mathrm{SD}=38$ $\mathrm{nm})$. The median size of Au-tpfc NPs was $83 \mathrm{~nm}(\mathrm{SD}=23 \mathrm{~nm}$ ) (Figures 1 and S5). Hydrodynamic and core size distributions were also verified by DLS and TEM (Figures S6$7)$.

Concentration measurements (NTA) and metal content analyses by ICP-MS were used to determine that both Au-tpfc and Ga-tpfc NPs in 2\% DMSO or 2\% pyridine housed $2.1 \times 10^{5}$ to $6.8 \times 10^{5}$ corroles/particle (Table S1). 


\subsection{Electronic Spectra.}

The electronic absorption spectra of Au-tpfc, Ga-tpfc(py), and Al-tpfc(py) $)_{2}$ in organic solvents and in aqueous media are shown in Figure 2. Note the sharp Soret absorption peaks attributable to monomeric corroles in organic solvents (black trace-pyridine or DMSO). Upon dispersion of corroles in aqueous media (red trace-2\% pyridine or $2 \%$ DMSO), the absorption bands broadened, indicating formation of NPs. Importantly, NP absorption maxima were very near those of monomers, suggesting that corrole-corrole electronic couplings are relatively weak. Our observation of similar peak locations in the absorption spectra of corrole monomers and corresponding NPs is consistent with Raman data (vide infra), further supporting our hypothesis of weak corrole-corrole NP electronic couplings. These findings also are in accord with those reported for some (but not all ${ }^{6,7}$ ) porphyrin NPs. ${ }^{13}$

\subsection{IR and Raman Spectra.}

The Raman vibrational features attributable to Au-tpfc monomers in pyridine solution are strikingly similar to those of NPs in $2 \%$ pyridine or in crystalline samples (Figure 3). Additional Raman spectra of NPs in aqueous DMSO solutions are in Figure S10. The conclusion is clear: intermolecular electronic interactions among Au-tpfc units in the crystal (or in NPs) are relatively weak: the closest ring-ring contacts are 3.4-3.5 $\AA$ in crystalline Au-tpfc (Figure S1); these contacts likely are similar in the NPs. The finding that Au-tpfc Soret absorptions are broadened in $2 \%$ pyridine suggests that there are many different corrole nanocluster arrangements in the NPs.

We have made assignments of the IR vibrational features using density functional theory (DFT). A comparison between the experimental and DFT-calculated IR spectrum of Au-tpfc is shown in Figure 4. The highest vibrational modes between 1600 and $1650 \mathrm{~cm}^{-1}$ in the IR spectrum of Au-tpfc are attributable to C-C bond stretching within the pentafluorophenyl rings; and the bands between 1300 and $1500 \mathrm{~cm}^{-1}$ can be assigned (DFT) to C-C and C-N bond stretching motions. From the DFT analysis, C-F bond stretching vibrations are below $1300 \mathrm{~cm}^{-1}$.

Experimental and DFT-calculated IR spectra for Al-tpfc are shown in Figure 4. The $620 \mathrm{~cm}$

${ }^{-1}$ band is attributable to axial Al-N(pyridine) vibrational motions (DFT calculated at 615 $\mathrm{cm}^{-1}$ ). As this band is absent in the Au-tpfc IR spectrum, we conclude that it is a spectroscopic signature of axially bound pyridine. Additional IR and Raman spectra of Au-, Al-, and Ga-tpfc NPs, crystals, and monomers are shown in Figures S11-S14; free base tpfc also forms NPs in 2\% DMSO (aqueous) media (Raman spectrum in Figure S15).

Raman spectra of Ga-tpfc in 2\% pyridine and in a crystal are shown in Figure 5. The spectrum in $2 \%$ pyridine is virtually identical with that of the crystal, indicating that pyridine remains axially coordinated in the NP. Comparisons of Al-tpfc spectra with 2\% DMSO, 2\% DMSO/2\% pyridine, and $2 \%$ pyridine also confirm binding of pyridine to the axial site (Figure 6). The peak corresponding to pyridine stretching appears at $620 \mathrm{~cm}^{-1}$ (black and red traces) and remains unchanged from $2 \%$ DMSO/2\% pyridine to $2 \%$ pyridine (Figure 7 ). 


\section{DISCUSSION}

\subsection{NP Structures.}

The spherical pyridine-solubilized NPs (Figures S6-S8) are structurally similar to the cores of protein-coated metallocorrole "sea urchin" aggregates. ${ }^{1}$ We suggest that weakly coupled corrole nanoclusters in Au-tpfc NPs have card-stacking arrangements (Figures 8a-c) similar to those in a crystal (Figure S16). These structures consist of parallel layers of corroles in which each layer is orthogonal to the next one (herringbone motif). In between each layer are small electrostatic cages, surrounded by pentafluorophenyl groups, with solvent bound inside (Figure 9).

We compare the card-stacking arrangement in Au-tpfc NPs to that of Ga-tpfc-pyridine (Figures $8 \mathrm{~d}-\mathrm{f}$ ). ${ }^{9}$ When an axial ligand is present, corroles can no longer $\pi$-stack. However, there are still layers of parallel corroles offset by approximately $45^{\circ}$. We expect the structures of Ga-tpfc aggregates to be similar to those in crystalline Ga-tpfc-pyridine. The size distribution of Al-tpfc NPs in 2\% pyridine is much smaller than that of any one of the other NPs. As we have no explanation for this finding, we will not speculate on corrolecorrole geometric arrangements in Al-tpfc NPs, although we have established that the intermolecular couplings are as weak (or weaker) than those in Au-tpfc and Ga-tpfc NPs.

As the solvent molecules will be trapped within these cages, we can construct a simple model to predict which solvents will prefer to bind to the corroles based on their gas-phase binding energies to pentafluorotoluene (mimicking one wall of the electrostatic cage). The binding energy of DMSO to pentafluorotoluene is $8.32 \mathrm{kcal} / \mathrm{mol}$, compared to $5.81 \mathrm{kcal} / \mathrm{mol}$ for water (structures optimized and energies calculated with PBE-D3/6-311G3DF-3PD), suggesting that corrole nanoparticles would prefer to incorporate DMSO over water. We also predict the ordering of solvent molecules on the surface of corrole nanoparticles based on their gas-phase binding energies to the center face of the corroles (Table S3). We find that the corrole face prefers to bind O-donor DMSO over both S-donor DMSO and water. We expect that the binding energy of O-donor DMSO to Ga-tpfc will be close to that of axially bound pyridine on the surface of the nanoparticle.

\section{CONCLUDING REMARKS}

Remarkably stable metallocorrole NPs can be made by dilution of DMSO or pyridine solutions of the corresponding monomers in aqueous media. NP corrole-corrole electronic couplings are evidenced by distinct broadening of Soret absorptions, although these couplings must be relatively weak, as Raman spectra of the quasi-soluble NPs are very similar to those of solvated monomers. We suggest that gold corrole NPs feature local regions with card stacking structures not unlike those in single crystals. The roles of DMSO and pyridine in solubilizing NPs are matters that require further study, as understanding these factors will surely be important in efforts to optimize the delivery of corroles to cancerous cells. 


\section{MATERIALS AND METHODS}

\subsection{NP Preparation and Characterization.}

Colloidal samples of Au-tpfc, Ga-tpfc(py), and Al-tpfc(py) $)_{2}$ were prepared by mixing metalcorrole dissolved in DMSO or pyridine into water. In a typical preparation a $20 \mu \mathrm{L}$ volume of metal-corrole stock solution $(\sim 4 \mathrm{mM})$ was added under vigorous stirring to $980 \mu \mathrm{L}$ of deionized water (in a glass vial) using a $50 \mu \mathrm{L}$ Hamilton syringe with the needle tip placed close to the micro stirring bar. Standard magnetic stirrer plates were used. No sign of precipitation was observed (Figures S2-4).

UV-visible absorption spectra were measured on a Ultraspec 3000 pro (GE Lifesciences). Particle size and concentration measurements were performed using a Malvern NanoSight NS300 instrument equipped with a 488-nm laser source and a syringe pump. Nanoparticle tracking analysis (NTA) is a technology for sizing particles in the 20 to $1000 \mathrm{~nm}$ size range. NTA combines laser light scattering microscopy with a CCD camera, which enables visualization of NPs dispersed in solvents. The NTA software tracks Brownian motion of individual particles and relates these motions to particle size. ${ }^{16}$ Importantly, the NanoSight software approximates each particle as a sphere and reports the hydrodynamic size distribution. Dynamic light scattering (DLS) measurements were conducted on a ZetaPALS instrument (Brookhaven Instruments Corporation).

NP metallocorrole content was determined by analyzing the same batch of NPs by NTA and inductively coupled plasma mass spectrometry (ICP-MS; details in SI). NTA was used to measure particle concentration, and ICP-MS was used to determine the metal content (ratio of 1:1 for metal/corrole). Calibration curves of external metal standards and calculations used to determine the number of metallocorroles per NP, using paired ICP-MS and NTA, are given in the Supporting Information (Table S1; Figure S17).

Transmission electron microscopy (TEM) images were taken on a FEI Tecnai 12 microscope equipped with a Gatan Ultrascan 2K CCD camera. Au-tpfc, Ga-tpfc-pyridine, and Al-tpfc(pyridine) 2 NPs were deposited on glow-discharged, carbon-coated 200 mesh Cu EM grids. Deposition was achieved by soaking the grids in NPs in $2 \%$ pyridine/water followed by removal and drying under a dinitrogen stream.

\subsection{Raman Spectroscopy.}

Liquid and solid samples for Raman spectroscopy confined in 1.5-mm ID glass capillary tubes (Fisher and PYREX brands) were irradiated at a $65^{\circ}$ angle of incidence with the 488 $\mathrm{nm}$ line from an Ar-ion laser (Coherent INNOVA 70). Radiation scattered around $0^{\circ}$ was collected (f/2) using a $100 \mathrm{~mm}$ Cannon macro camera lens, filtered with a 488-nm sharp edge long pass filter, focused onto the entrance slit of a Spex $0.75 \mathrm{~m}$ spectrograph, and detected using a thermoelectrically cooled CCD camera (Horiba Synapse). Data acquisition, controlled by Horiba SynerJY software, involved multiple 1-s exposures averaged to achieve acceptable signal-to-noise levels. 


\subsection{IR Spectroscopy.}

Potassium bromide $(\mathrm{KBr})$ pellets of solid samples were prepared and each confined in a 13$\mathrm{mm}$ stainless steel hydraulic die (PIKE technology). Just before mounting the die in the nitrogen purged compartment of a FTIR spectrometer (BRUKER VERTEX 80, OPUS software) with a liquid nitrogen cooled detector, a background scan in a nitrogen environment was taken; subsequently, the die was mounted and a 16-scan sample spectrum was taken in transmittance mode.

\subsection{DFT Calculations.}

All quantum mechanics computations were performed using density functional theory (DFT) with either the PBE0 ${ }^{17}$ functional (for vibrational spectra) or $\mathrm{PBE}^{18}$ (for energy calculations) functional with Grimme D3 dispersion corrections ${ }^{19,20}$ when necessary, implemented in the Schrödinger Jaguar Software. ${ }^{21}$ The basis sets are noted as used, including the $6-311 \mathrm{G}^{* *++}$ extended basis set ${ }^{22-25}$ (including diffuse and polarization functions), and the Los Alamos effective core potentials LACV3P**++ (based on the 6$311 \mathrm{G}$ basis) and LACVP (based on the 6-31G basis) basis sets. ${ }^{26}$ An implicit solvation model, $\mathrm{PBF},{ }^{27,28}$ was used for all vibrational calculations with a dielectric constant mimicking that of water (80.37).

\section{Supplementary Material}

Refer to Web version on PubMed Central for supplementary material.

\section{ACKNOWLEDGMENTS}

We thank Zhuo Li (City of Hope Electron Microscopy Core Facility) for expert help with TEM experiments. Raman and IR spectra were collected (by MHBG) in the Beckman Institute Laser Resource Center; and time on the computing cluster (for JO) was provided by the Beckman Institute Materials and Process Simulation Center. The Arnold and Mabel Beckman Foundation supports the BI Laser and Materials Centers.

Funding Sources

Research at Caltech was supported by the National Institutes of Health (DK-019038 to HBG) and by an Ernest H. Swift Summer Undergraduate Research Fellowship (to JO). Research at the City of Hope (JT) and the Technion (ZG) was supported by an International Collaboration Grant from the Jacki and Bruce Barron Cancer Research Scholars Program, a partnership of the ICRF and City of Hope funded by the Harvey L. Miller Family Foundation.

\section{REFERENCES}

(1). Soll M; Goswami TK; Chen Q-C; Saltsman I; Teo RD; Shahgholi M; Lim P; Di Bilio AJ; Cohen S; Termini J; et al. Cell-Penetrating Protein/Corrole Nanoparticles. Scientific Reports 2019, 9 (1), 2294. [PubMed: 30783138]

(2). Rideal EK Discussion on Surface Phenomena-Films. Proc. R. Soc. Lond. A 1936, 155 (886), 684-711. 10.1098/rspa.1936.0128.

(3). Alexander A 381. Monolayers of Porphyrins and Related Compounds. Journal of the Chemical Society (Resumed) 1937, 1813-1816.

(4). Bergeron JA; Gaines GL; Bellamy WD Monolayers of Porphyrin Esters: Spectral Disturbances and Molecular Interactions. Journal of Colloid and Interface Science 1967, 25 (1), 97-106. 10.1016/0021-9797(67)90014-8.

(5). White WI Aggregation of Porphyrins and Metalloporphyrins. The Porphyrins 1978, 5, 303-339. 
(6). Pasternack RF; Schaefer KF; Hambright P Resonance Light-Scattering Studies of Porphyrin Diacid Aggregates. Inorganic Chemistry 1994, 33 (9), 2062-2065.

(7). Würthner F; Kaiser TE; Saha-Möller CR J-Aggregates: From Serendipitous Discovery to Supramolecular Engineering of Functional Dye Materials. Angewandte Chemie International Edition 2011, 50 (15), 3376-3410. [PubMed: 21442690]

(8). Soll M; Sudhakar K; Fridman N; Müller A; Röder B; Gross Z One-Pot Conversion of Fluorophores to Phosphorophores. Org. Lett 2016, 18 (22), 5840-5843. 10.1021/acs.orglett. 6b02877. [PubMed: 27934504]

(9). Bendix J; Dmochowski IJ; Gray HB; Mahammed A; Simkhovich L; Gross Z Structural, Electrochemical, and Photophysical Properties of Gallium(III) 5,10,15Tris(Pentafluorophenyl)Corrole. Angewandte Chemie International Edition 2000, 39 (22), 4048 4051. 10.1002/1521-3773(20001117)39:22<4048::AID-ANIE4048>3.0.CO;2-7. [PubMed: 11093200]

(10). Mahammed A; Gross Z Aluminum Corrolin, a Novel Chlorophyll Analogue. Journal of Inorganic Biochemistry 2002, 88 (3), 305-309. 10.1016/S0162-0134(01)00373-7. [PubMed: 11897344]

(11). Teo RD; Hwang JY; Termini J; Gross Z; Gray HB Fighting Cancer with Corroles. Chemical Reviews 2016, 117 (4), 2711-2729. [PubMed: 27759377]

(12). Zou T; Lum CT; Lok C-N; Zhang J-J; Che C-M Chemical Biology of Anticancer Gold (III) and Gold (I) Complexes. Chemical Society Reviews 2015, 44 (24), 8786-8801. [PubMed: 25868756]

(13). Drain CM; Smeureanu G; Patel S; Gong X; Garno J; Arijeloye J Porphyrin Nanoparticles as Supramolecular Systems. New Journal of Chemistry 2006, 30 (12), 1834-1843.

(14). Crucho CI; Barros MT Polymeric Nanoparticles: A Study on the Preparation Variables and Characterization Methods. Materials Science and Engineering: C 2017, 80, 771-784. [PubMed: 28866227]

(15). Pasternack RF; Collings PJ Resonance Light Scattering: A New Technique for Studying Chromophore Aggregation. Science 1995, 269 (5226), 935-939. [PubMed: 7638615]

(16). Filipe V; Hawe A; Jiskoot W Critical Evaluation of Nanoparticle Tracking Analysis (NTA) by NanoSight for the Measurement of Nanoparticles and Protein Aggregates. Pharmaceutical research 2010, 27 (5), 796-810. [PubMed: 20204471]

(17). Adamo C; Barone V Toward Reliable Density Functional Methods without Adjustable Parameters: The PBE0 Model. The Journal of Chemical Physics 1999, 110 (13), 6158-6170.

(18). Perdew JP; Burke K; Ernzerhof M Generalized Gradient Approximation Made Simple. Physical Review Letters 1996, 77 (18), 3865. [PubMed: 10062328]

(19). Grimme S; Antony J; Ehrlich S; Krieg H A Consistent and Accurate Ab Initio Parametrization of Density Functional Dispersion Correction (DFT-D) for the 94 Elements H-Pu. The Journal of Chemical Physics 2010, 132 (15), 154104. [PubMed: 20423165]

(20). Goerigk L; Grimme S A Thorough Benchmark of Density Functional Methods for General Main Group Thermochemistry, Kinetics, and Noncovalent Interactions. Physical Chemistry Chemical Physics 2011, 13 (14), 6670-6688. [PubMed: 21384027]

(21). Bochevarov AD; Harder E; Hughes TF; Greenwood JR; Braden DA; Philipp DM; Rinaldo D; Halls MD; Zhang J; Friesner RA Jaguar: A High-Performance Quantum Chemistry Software Program with Strengths in Life and Materials Sciences. Int. J. Quantum Chem 2013, 113 (18), 2110-2142. 10.1002/qua.24481.

(22). Clark T; Chandrasekhar J; Spitznagel GW; Schleyer PVR Efficient Diffuse Function-Augmented Basis Sets for Anion Calculations. III. The 3-21+G Basis Set for First-Row Elements, Li-F. Journal of Computational Chemistry 4 (3), 294-301. 10.1002/jcc.540040303.

(23). Frisch MJ; Pople JA; Binkley JS Self-Consistent Molecular Orbital Methods 25. Supplementary Functions for Gaussian Basis Sets. The Journal of Chemical Physics 1984, 80 (7), 3265-3269.

(24). Krishnan R; Binkley JS; Seeger R; Pople JA Self-consistent Molecular Orbital Methods. XX. A Basis Set for Correlated Wave Functions. The Journal of Chemical Physics 1980, 72 (1), 650654. 10.1063/1.438955.

(25). McLean AD; Chandler GS Contracted Gaussian Basis Sets for Molecular Calculations. I. Second Row Atoms, Z=11-18. The Journal of Chemical Physics 1980, 72 (10), 5639-5648. 10.1063/1.438980. 
(26). Hay PJ; Wadt WR Ab Initio Effective Core Potentials for Molecular Calculations. Potentials for $\mathrm{K}$ to Au Including the Outermost Core Orbitals. The Journal of Chemical Physics 1985, 82 (1), 299-310.

(27). Tannor DJ; Marten B; Murphy R; Friesner RA; Sitkoff D; Nicholls A; Honig B; Ringnalda M; Goddard WA III Accurate First Principles Calculation of Molecular Charge Distributions and Solvation Energies from Ab Initio Quantum Mechanics and Continuum Dielectric Theory. Journal of the American Chemical Society 1994, 116 (26), 11875-11882.

(28). Marten B; Kim K; Cortis C; Friesner RA; Murphy RB; Ringnalda MN; Sitkoff D; Honig B New Model for Calculation of Solvation Free Energies: Correction of Self-Consistent Reaction Field Continuum Dielectric Theory for Short-Range Hydrogen-Bonding Effects. The Journal of Physical Chemistry 1996, 100 (28), 11775-11788. 

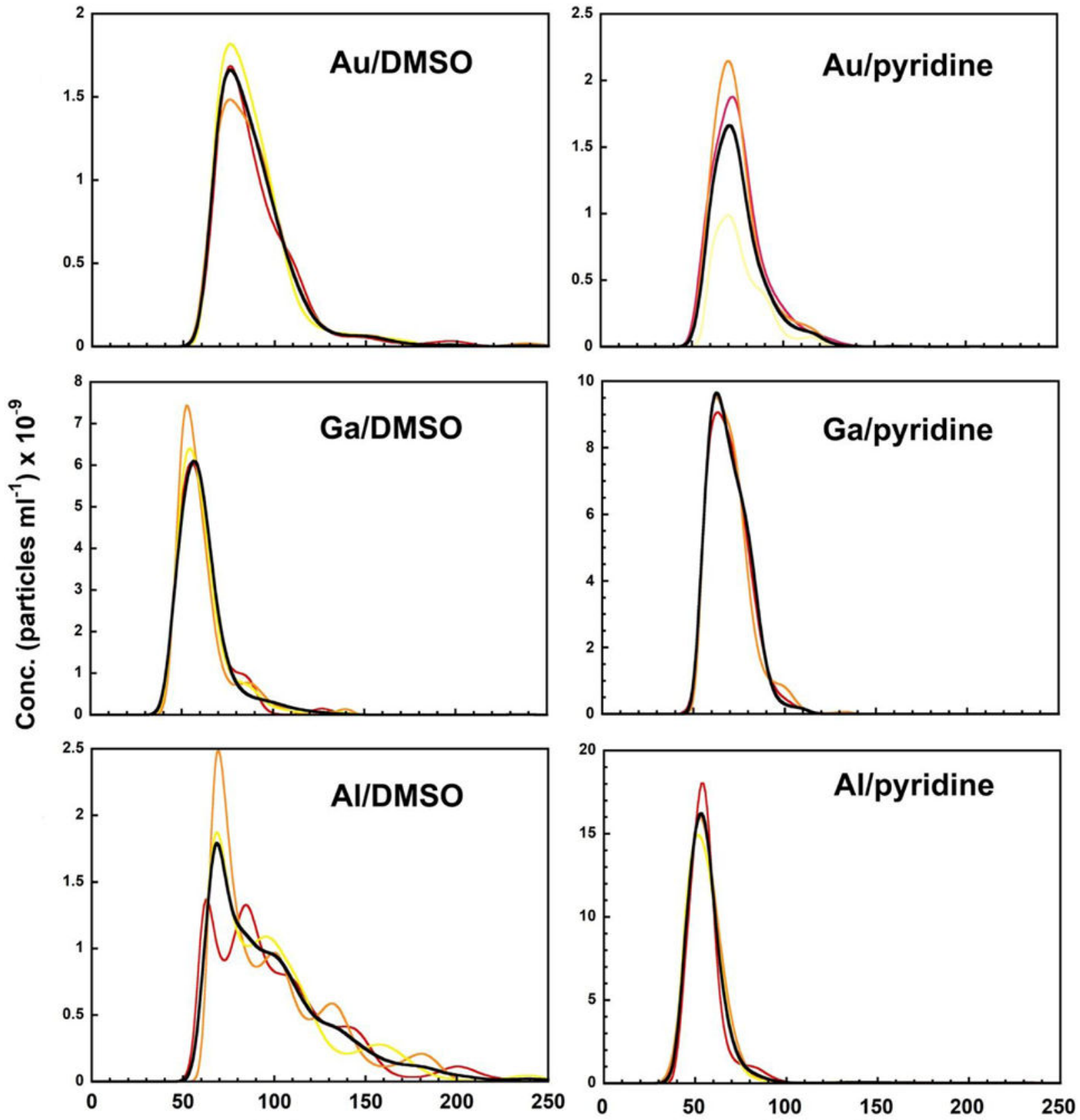

Diameter (nm)

Figure 1.

Average particle size distributions (black traces) for Au-tpfc, Ga-tpfc(py), and Al-tpfc(py) 2 $\mathrm{NP}$ samples in $2 \% \mathrm{DMSO} /$ water (left) and $2 \%$ pyridine/water (right) measured at $25^{\circ} \mathrm{C}$ (see Material and Methods). Averages are based on three captures (colored traces, see Figures S5.1-5.3 for experimental and statistical parameters). 

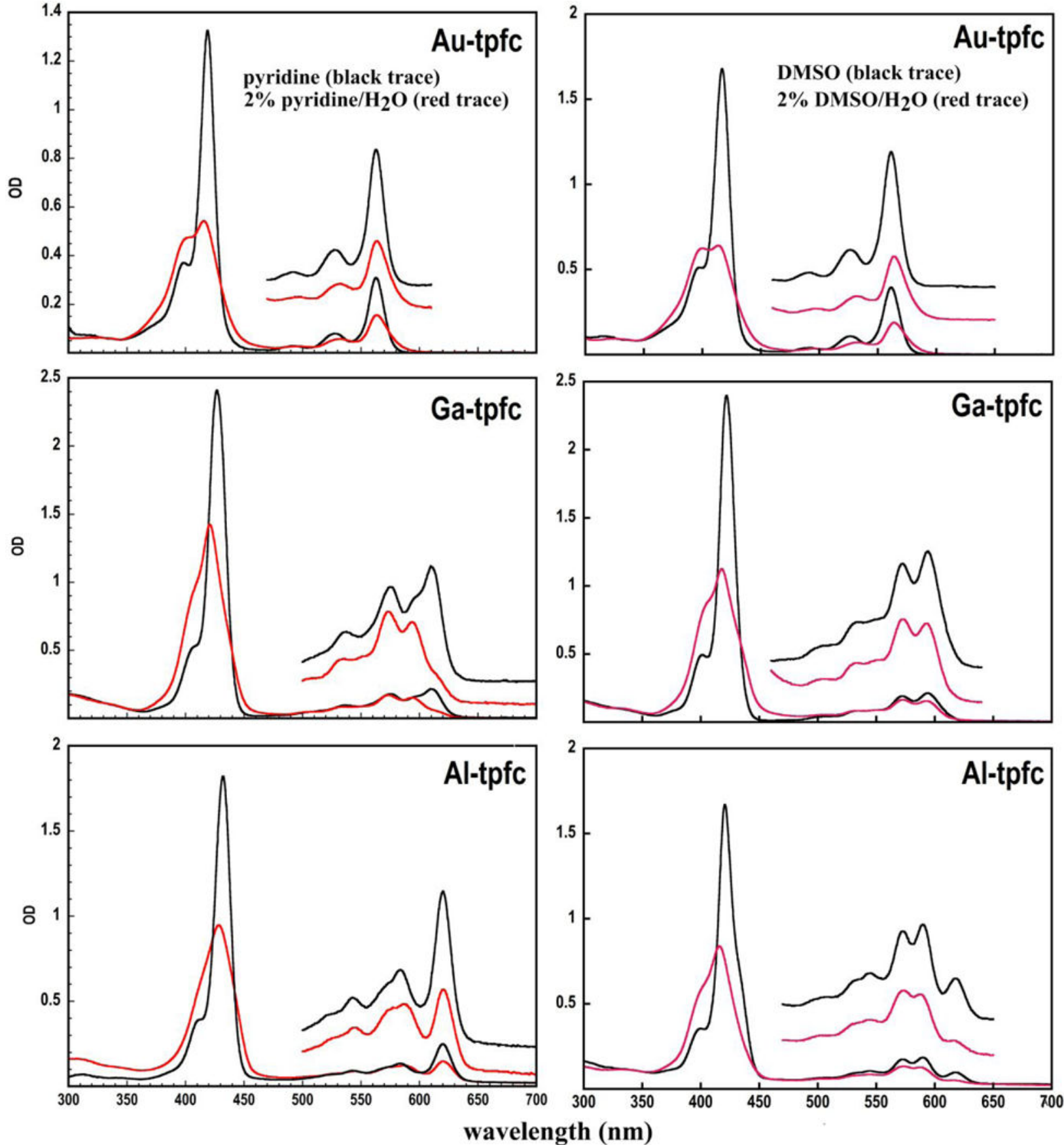

Figure 2.

Electronic absorption spectra of $\mathrm{M}(\mathrm{III})$-tpfc $(\mathrm{M}=\mathrm{Au}, \mathrm{Ga}, \mathrm{Al})$ in pure pyridine and $2 \%$ pyridine/water (left column); and in pure DMSO and 2\% DMSO/water (right column). Monomeric samples contained $20 \mu \mathrm{L}$ of fresh metal-corrole stock solution ( $4 \mathrm{mM}$ in pure pyridine or DMSO) diluted in $980 \mu \mathrm{L}$ of organic solvent. NP samples were prepared sequentially using the same amount of stock corrole solution for proper comparisons (see Material and Methods section). 1-mm path-length quartz cuvettes were used for optical density (OD) measurements (see Figure S9 for extinction coefficients). It is noteworthy that 
the values of the integrated areas of the Soret band were roughly the same for both samples in each set. 


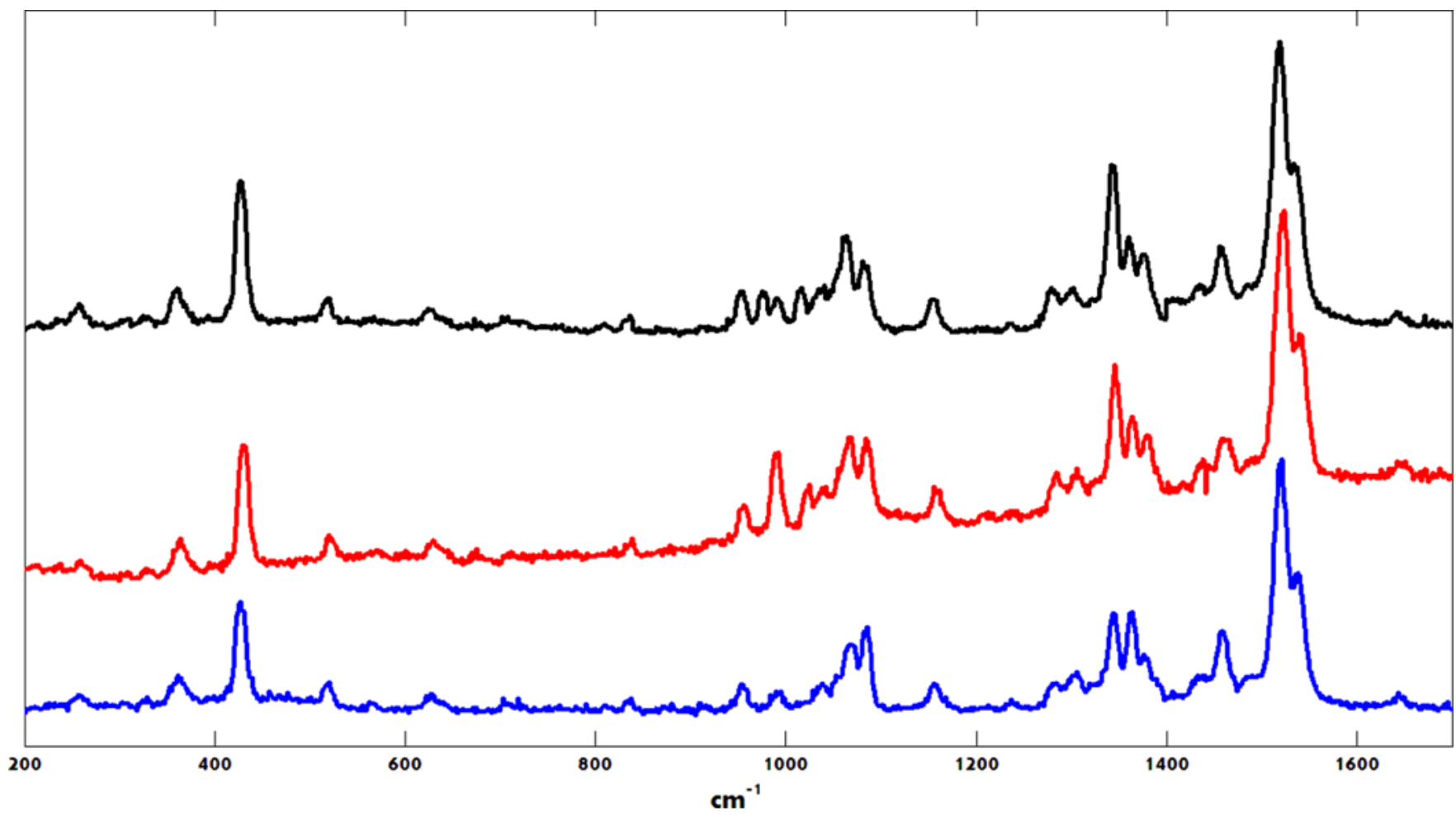

Figure 3.

Raman spectra: Au-tpfc in pure pyridine (black); in $2 \%$ pyridine (red); crystalline sample (blue); $457.9 \mathrm{~nm}$ excitation. 


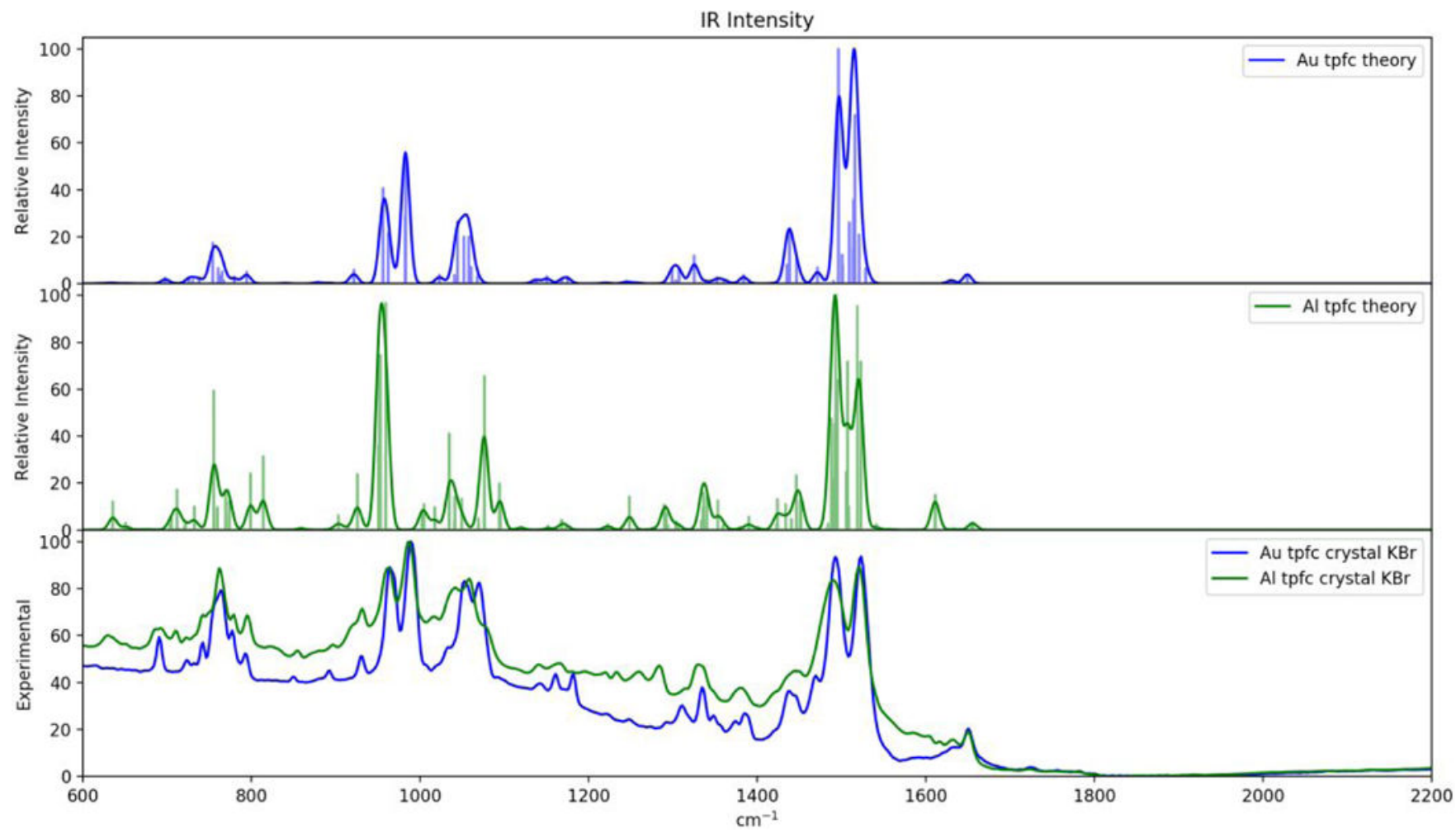

Figure 4.

Top panel: IR spectrum of Au-tpfc calculated using PBE0/LACV3P** with a 0.96 vibrational shift and gaussian smoothing (standard deviation of $5 \mathrm{~cm}^{-1}$ ) (blue). Middle panel: IR spectrum of Al-tpfc with two axial pyridines calculated using PBE0/6-31G with a 0.96 vibrational shift and gaussian smoothing (standard deviation of $5 \mathrm{~cm}^{-1}$ ) (green). Bottom panel: Experimental IR spectrum of crystalline Au-tpfc (blue) and crystalline Altpfc (green). The axial ligand [Al-N(py)] vibrational signature is at $620 \mathrm{~cm}^{-1}$. 


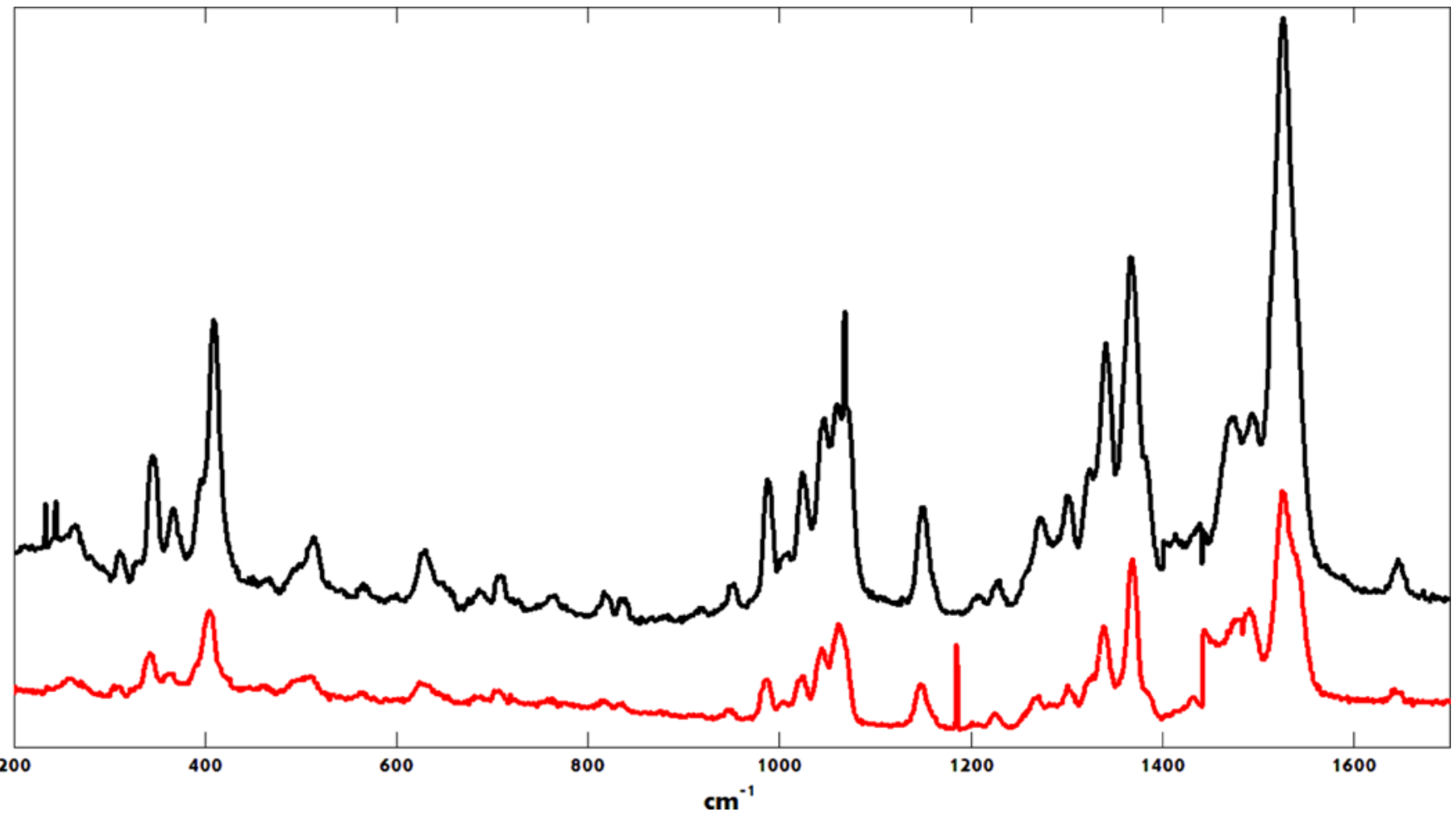

Figure 5.

Raman spectra: Ga-tpfc in 2\% pyridine (black); crystalline sample (red); $457.9 \mathrm{~nm}$ excitation. The spikes just below $1200 \mathrm{~cm}^{-1}$ in the red curve and at $220 \mathrm{~cm}^{-1}$ in the black curve are attributable to cosmic ray events. 


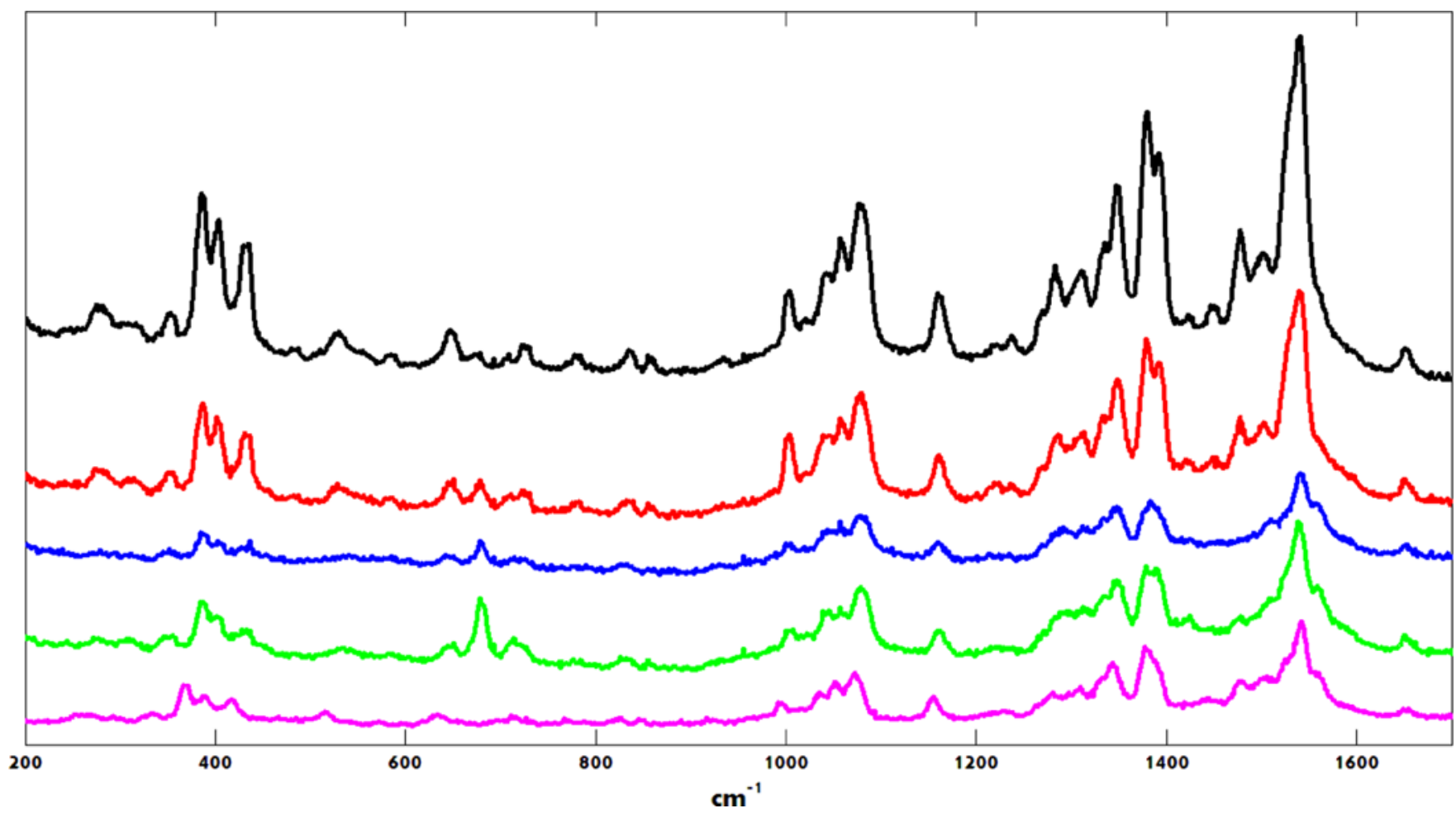

Figure 6.

Raman spectra of Al-tpfc(py) $2: 2 \%$ py NPs (black); $2 \% \mathrm{DMSO} / 2 \%$ py NPs (red); $2 \%$ DMSO NPs (blue); 5\% DMSO NPs (green); crystalline sample (magenta); $457.9 \mathrm{~nm}$ excitation. 


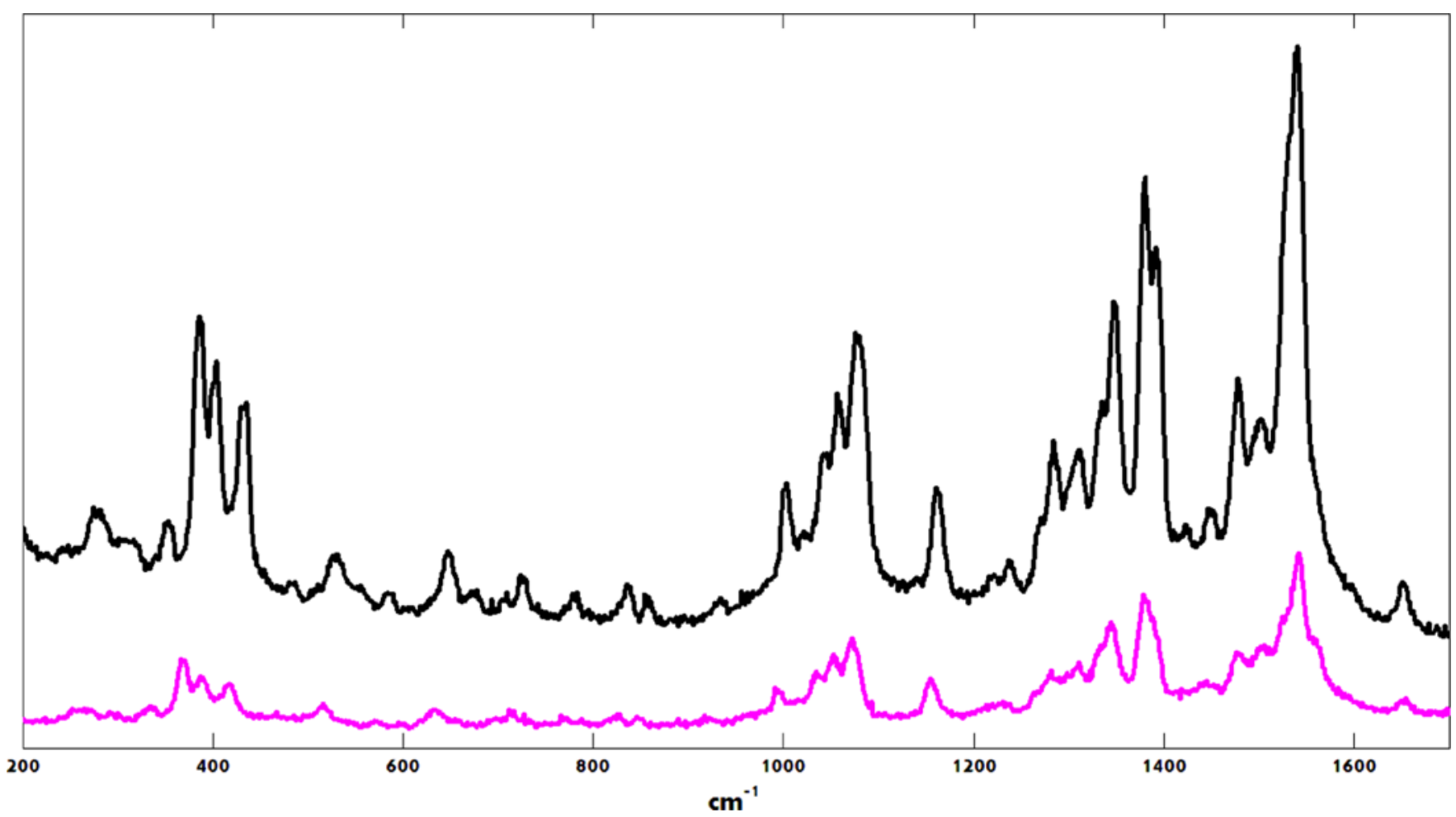

Figure 7.

Raman spectra of Al-tpfc(py)2: $\%$ py NPs (black); crystal (magenta); $457.9 \mathrm{~nm}$ excitation. 
a)

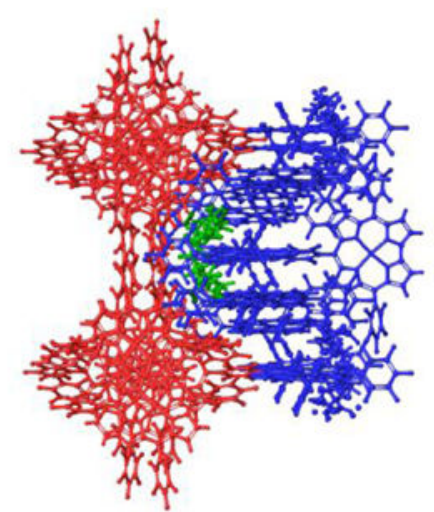

b)

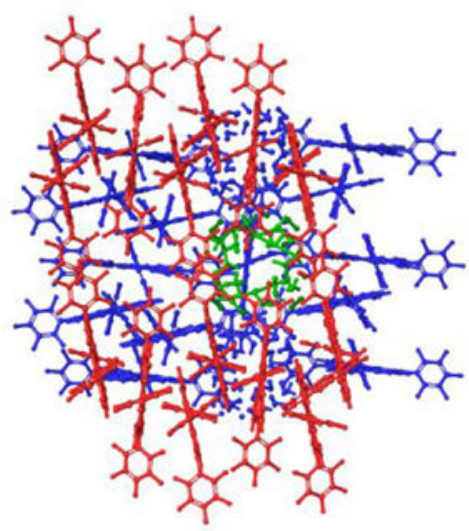

c)

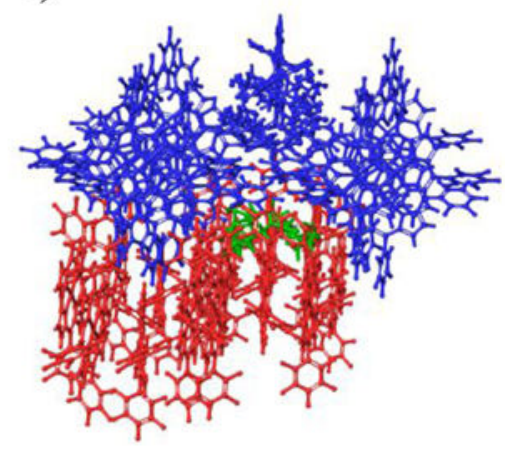

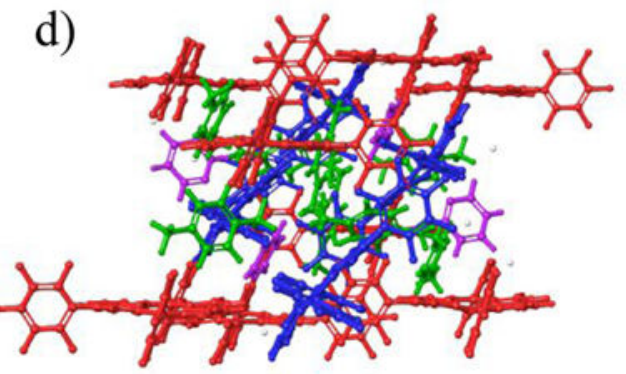

e)
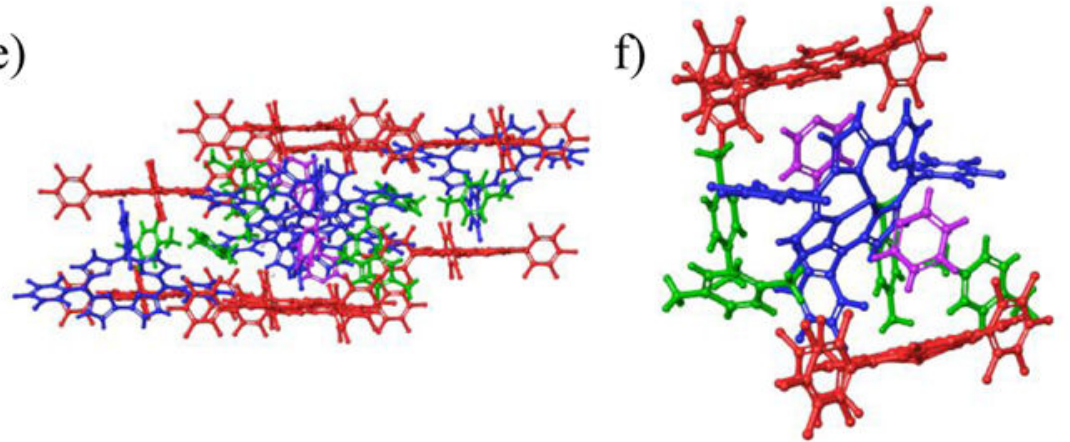

Figure 8.

Proposed card-stacking Au-tpfc nanocluster structures (a-c) based on the arrangements of corroles in crystals (Figure S16). Proposed Ga-tpfc nanocluster structures (d-f) based on Gacorrole crystal packing (see ref. 9). The red and blue molecules represent two layers of $\mathrm{Au}-$ tpfc or Ga-tpfc corroles, the green molecules depict solvent molecules (p-xylene), axial pyridines are purple. 
a)

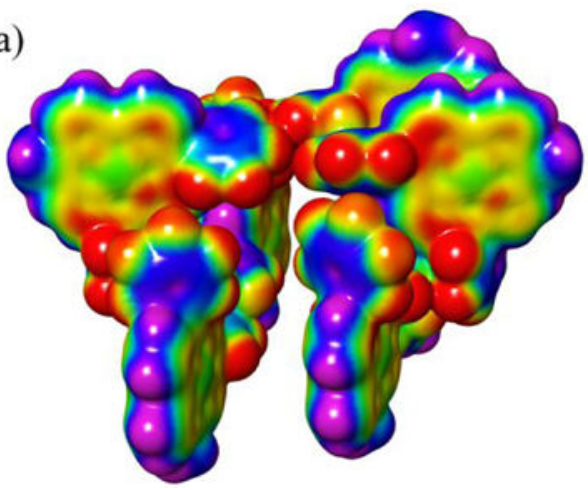

b)

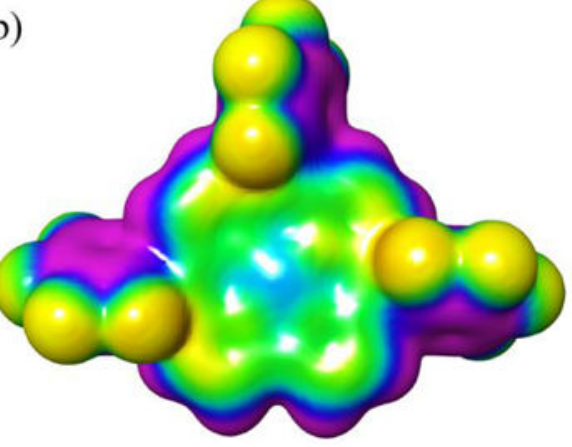

c)

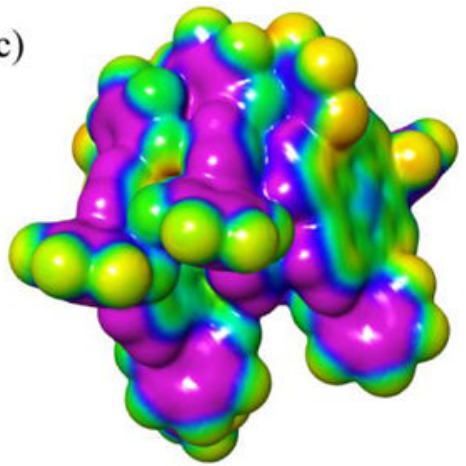

Figure 9.

PBE-D3/LACVP calculated electron density diagrams with electrostatic potentials mapped on the crystal substructures. (a) Cage like structure, (b) monomer, and (c) stacked tetramer. 